\title{
Enhancing Epidemic Detection Using Syndromic Surveillance and Early Notification Methods
}

\author{
Tippa Wongstitwilairoong*1, Saranath Lawpoolsri Niyom ${ }^{1}$, \\ Ngamphol Soonthornworasiri ${ }^{1}$, Jariyanart Gaywee ${ }^{2}$ and Jaranit Kaewkungwal ${ }^{1}$
}

${ }^{1}$ Faculty of Tropical Medicine, Mahidol University, Bangkok, Thailand; ${ }^{2}$ Royal Thai Army-Armed Forces Research Institute of Medical Sciences, Bangkok, Thailand

\section{Objective}

This paper presents an investigation using early notification methods to enhancing epidemic detection in syndromic surveillance data from royal Thai army in Thailand.

\section{Introduction}

Early Notification Detection Systems have taken a critical role in providing early notice of disease outbreaks. To improve the detection methods for disease outbreaks, many detection methods have been created and implemented. However, there is limited information on the effectively of syndromic surveillance in Thailand. Knowing the performance, strengths and weakness of these surveillance systems in providing early warning for outbreaks will increase disease outbreak detection capacity in Thailand.

\section{Methods}

This study describes and compares the capabilities of various outbreak detection algorithms using 37,043 unique syndromic daily reports based on medical information from both civilian and military personnel from the Unit Base Surveillance of Royal Thai Army (RTA) along the Thai-Myanmar and Thai-Cambodia boarder areas. Traditional epidemic detection method: mean plus two SD were compared with algorithms for early notification methods and which included regression, regression/EWMA/Poisson, CDC-C1, CDC-C2 and CDC-C3. Early notification and epidemic detection methods were compared according to their ability to generate alert notifications. Sensitivity, specificity, positive predictive value (PPV), negative predictive value and overall accuracy to detect or predict disease outbreaks were estimated.

\section{Results}

This study shows that the preliminary results are promising for epidemic detection by early notification methods in syndromic surveillance in Thailand. The majority of syndromic records were categorized into 12 symptoms. The three most common symptoms were respiratory, fever and gastrointestinal illness $(11,501 ; 9,549$ and 4,498 respectively). The results from the early notification systems were analyzed and their performances were compared with traditional epidemic detection method according to their ability to generate early warning alerts for the 3 symptoms. In our study regression/EWMA/ Poisson method had higher specificity across the 3 symptoms $(94.5 \%$, $94.7 \%$ and $95.9 \%$ respectively), but generated lower sensitivity (22.6\%, 40.4\% and 23.1\%). CDC-C1, CDC-C2 and CDC-C3 algorithms are easy to understand and are widely used. CDC-C3 had higher sensitivity to detect gradual disease outbreak effects $(64.2 \%$, $70.2 \%$ and $57.7 \%$ ), but it is known to produce higher alarm rates/false positive signals.

\section{Conclusions}

Within the syndromic surveillance data of RTA, the CDC algorithm is the best chosen to use in the syndromic system due to being easy to understand and implement in a system with high sensitivity. CDC-C2 is the best early notification detection method due to its high sensitivity and PPV. However, CDC-C3 is shows the highest sensitivity, but exhibits the lowest specificity and PPV for all symptoms including a high alarm rates. To be useful, early notification detection methods must have acceptable operating characteristics. Consequently, we should select the most appropriate algorithm method to explain the data well and in order to improve detection of outbreaks. The comparison methods used in this study may be useful for testing other proposed alert threshold methods and may have further applications for other populations and other diseases.

\section{Keywords}

early notification; epidemic detection; syndromic

\section{Acknowledgments}

Great appreciation to all study site health workers, who coordinated, and collected data.

\section{References}

1. Chretien JP, Burkom HS, Sedyaningsih ER, Larasati RP, et al. Syndromic Surveillance: Adapting Innovations to Developing Settings. PLoS Medicine 2008; vol 5: page 1-6.

2. Burkom HS, Elbert Y, Magruder SF, Najmi AH, Peter W, Thompson MW. Developments in the roles, features, and evaluation of alerting algorithms for disease outbreak monitoring. Johns Hopkins APL Technical Digest 2008; vol 27: page 313.

*Tippa Wongstitwilairoong

E-mail: tippaw@afrims.org 